\title{
Cosmic rays and Radio Halos in galaxy clusters : new constraints from radio observations
}

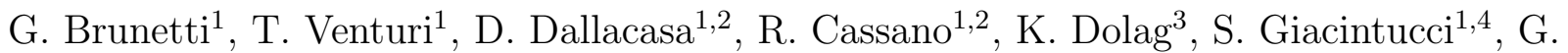 \\ Setti ${ }^{1,2}$
}

\begin{abstract}
Clusters of galaxies are sites of acceleration of charged particles and sources of non-thermal radiation. We report on new constraints on the population of cosmic rays in the Intra Cluster Medium (ICM) obtained via radio observations of a fairly large sample of massive, $\mathrm{X}$-ray luminous, galaxy clusters in the redshift interval 0.2-0.4. The bulk of the observed galaxy clusters does not show any hint of Mpc scale synchrotron radio emission at the cluster center (Radio Halo). We obtained solid upper limits to the diffuse radio emission and discuss their implications for the models for the origin of Radio Halos. Our measurements allow us to derive also a limit to the content of cosmic ray protons in the ICM. Assuming spectral indices of these protons $\delta=2.1-2.4$ and $\mu \mathrm{G}$ level magnetic fields, as from Rotation Measures, these limits are one order of magnitude deeper than present EGRET upper limits, while they are less stringent for steeper spectra and lower magnetic fields.
\end{abstract}

Subject headings: particle acceleration - radiation mechanisms: non-thermal galaxies: clusters: general - radio continuum: general - X-rays: general

\section{Introduction}

Clusters of galaxies are ideal astrophysical environments for particle acceleration. Large scale shocks which form during the process of cluster formation are believed to be efficient particle accelerators (e.g. Sarazin 1999; Gabici \& Blasi 2003; Ryu et al. 2003; Pfrommer

\footnotetext{
${ }^{1}$ INAF/IRA, via Gobetti 101, I-40129 Bologna, Italy

${ }^{2}$ Dip. Astronomia, University of Bologna, via Ranzani 1, I-40127 Bologna, Italy

${ }^{3}$ Max-Planck-Institut für Astrophysik, Karl-Schwarzschild Strasse 1, D-85741 Garching bei München, Germany

${ }^{4}$ Harvard-Smithsonian Center for Astrophysics, 60 Garden Street, Cambridge, MA 02138
} 
et al. 2006). Cosmic rays (CRs) can also be injected into the ICM from ordinary galaxies and AGNs (e.g. Völk \& Atoyan 1999) and turbulent eddies may contribute to the particle acceleration process (e.g. Brunetti \& Lazarian 2007). CRs accelerated within the cluster volume would then be confined for cosmological times and the bulk of their energy is expected in protons since they have radiative and collisional life-times much longer than those of the electrons (e.g. Blasi et al. 2007, for a review).

While present gamma ray observations can only provide upper limits to the average energy density of CR protons in the ICM (e.g. Reimer et al. 2004), the presence of relativistic electrons in a number of clusters has been ascertained via the detection of a tenuous synchrotron radio emission: giant Radio Halos (RHs) and mini-Radio Halos, fairly symmetric sources at the cluster center, and Radio Relics, elongated sources at the cluster periphery (e.g. Feretti \& Giovannini 2007). It is customary to classify the models for the origin of RHs in secondary electron (e.g. Blasi \& Colafrancesco 1999) and reacceleration models (e.g. Brunetti et al. 2001; Petrosian 2001), depending on whether the radiating electrons are produced as secondary products of hadronic interactions or reaccelerated by turbulence from a pre-existing population of non-thermal seeds in the ICM, respectively. These models predict a different connection between radio and X-ray properties of clusters which are discussed in this Letter and compared with new observations: in Sect. 2 we review the expectations of the different models, in Sect.3 we briefly present the radio observations of our cluster sample, and in Sects. $4 \& 5$ we report and discuss our results. Concordance $\left(H_{o}=70, \Omega_{m}=0.3, \Omega_{\Lambda}=0.7\right)$ cosmology is used.

\section{Radio - X-ray correlation \& origin of RH}

Giant RHs follow a correlation between their radio power at $1.4 \mathrm{GHz}\left(P_{1.4}\right)$ and physical

size, and the X-ray luminosity $\left(L_{X}\right)$ and temperature of clusters in which they are found (e.g. Liang et al. 2000; Bacchi et al. 2003; Cassano et al. 2006,07).

In this Letter we focus on the $P_{1.4}-L_{X}$ correlation that relates directly observable quantities. The bulk of giant RHs has been discovered from the analysis of relatively shallow surveys (NVSS, Giovannini et al. 1999, G99; WENSS, Kempner \& Sarazin 2001, KS01) and a relevant issue is how observational biases may affect this correlation. There is agreement on the fact that the upper envelope of the $P_{1.4}-L_{X}$ correlation is likely to be solid (e.g. Clarke 2005), but the effect of observational biases on the lower envelope is more problematic (Rudnick et al. 2006). Indeed, if all clusters would have cluster-scale radio emission at the level of presently known RHs, then the $P_{1.4}-L_{X}$ trend may possess a fairly large spread with lower luminosity RHs falling just below present observational limits. On the other hand 
it is also possible that clusters have a physical bimodal distribution with the $\mathrm{RH}$-clusters following the correlation and with other clusters having no (or much weaker) cluster-scale radio emission.

The first possibility is essentially what secondary models would expect (e.g. Miniati et al. 2001; Dolag \& Ensslin 2000). This comes from the combination of two points: the magnetization at $\mu \mathrm{G}$ level is believed to be a very common property of the ICM (Clarke et al. 2001; Govoni \& Feretti 2004), and CR protons accumulated in galaxy clusters for cosmological time scales provide a fairly stable continuous source of injection of secondary electrons and positrons in the ICM.

On the other hand, an unavoidable prediction of the re-acceleration scenario is a bimodality of clusters. In this scenario particles are supposed to be re-accelerated by MHD turbulence in the ICM and this requires enough turbulence to boost electrons at the energies necessary to emit synchrotron radiation at GHz frequencies. Thus giant RHs should be strictly connected to massive and merging systems where indeed enough turbulence can be developed (Cassano \& Brunetti 2005), and should live for relatively short time-scales (1 Gyr or less) because of the finite dissipation time-scale of turbulence. In this scenario merging clusters are expected to move with time from a radio quiet region in the $P_{1.4}-L_{X}$ plane to the $P_{1.4}-L_{X}$ correlation. This happens in a relatively short time-scale, of the order of $\approx 10^{8} \mathrm{yrs}$ (i.e. the acceleration time-scale of the emitting particles, see Fig.19 in Brunetti et al. 2004), and thus the region between $\mathrm{RHs}$ and radio quiet clusters in the $P_{1.4}-L_{X}$ plane should be poorly populated.

Thus, the distribution of clusters in the $P_{1.4}-L_{X}$ plane is important to constrain current models.

\section{Cluster sample and GMRT observations}

From the ROSAT-ESO Flux Limited X-ray (REFLEX) galaxy cluster catalog (Böhringer et al. 2004) and from the extended ROSAT Brightest Cluster Sample (eBCS) catalog (Ebeling et al. 1998, 2000) we selected all clusters with $0.2 \leq z \leq 0.4, \mathrm{~L}_{\mathrm{X}}(0.1-2.4 \mathrm{keV})>5 \times$ $10^{44} \mathrm{erg} \mathrm{s}^{-1}$, and with declination $\delta \geq-30^{\circ}$ (for the REFLEX) and $15^{\circ}<\delta<60^{\circ}$ (for the eBCS). These selection criteria led to a sample of $50 \mathrm{X}$-ray selected galaxy clusters $(27$ from REFLEX, 23 from eBCS) with similar luminosity. The sample includes 6 clusters with well studied RHs (A 2744, A 1300, A 2163, A 773, A 2219, A 2390, e.g. Feretti \& Giovannini 2007; Bacchi et al. 2003) and A 1758 for which hint of diffuse emission is also reported (G99, KS01). 
We carried out GMRT (Giant Metrewave Radio Telescope) observations at $610 \mathrm{MHz}$ only for 34 clusters in the sample (those with no high sensitivity radio information already available, and not included in the GMRT cluster key project); each cluster was observed for 2-3 hrs (hour angle 3-5). Thanks to the dirtibution of antennas at GMRT we obtained images for each cluster with resolutions ranging from $\sim 6^{\prime \prime}$ to $\sim 25^{\prime \prime}$ and r.m.s. $(1 \sigma) \sim 30-180 \mu \mathrm{Jy} / \mathrm{b}$ (Venturi et al. 2007, V07; Venturi et al., in prep) which allows us to image both compact and extended sources in the fields. We detected RHs in 4 of them (RXCJ 2003-2323; A 209 and RXCJ 1314-2515, V07; A 697 Venturi et al. in prep.; a Relic was also found in A 521, Giacintucci et al. 2006). No hint of cluster-scale radio emission was found in the remaining 29 clusters. For these clusters it is necessary to place solid upper limits to the flux density of their Mpc scale radio emission (few arcmin at the redshifts of our clusters). In Fig. 1 we report the normalised integrated brightness profiles of well studied RHs: they are quite similar and $\approx 50 \%$ of the luminosity, $L_{H}$, is emitted within about half radius, $R_{H}$. Detection limit based on the brightness within $R_{H} / 2$ gives a $610 \mathrm{MHz}$ luminosity in $\mathrm{W} / \mathrm{Hz}\left(\theta_{b}\right.$ is the beam-FWHM):

$$
L_{H} \geq 3.5 \cdot 10^{23}\left(1+\frac{z}{0.25}\right)^{4} \frac{(\text { r.m.s. })}{50 \mu J y / b}\left(\frac{25^{\prime \prime}}{\theta_{b}}\right)^{2}\left(\frac{R_{H}}{0.5 M p c}\right)^{2}
$$

which is $\approx 50$ times smaller than luminosities of known giant RHs at $z \approx 0.25(\mathrm{~V} 07)$.

In order to derive more solid constraints to use in this paper we inject fake RHs in our datasets. We model the brightness profile in Fig. 1 with sets of optically thin spheres with different radius and flux densities, and obtain families of fake RHs with total flux densities $\mathrm{S}_{R H}$ ranging from 3 to $300 \mathrm{mJy}$ and angular diameters from 180 to 350 arcsec. Those fake RHs were injected into the uv-components of our cluster datasets by means of the task UVMOD in AIPS, and the resulting datasets were imaged with the procedures given in V07 with the task IMAGR at resolutions in the range 10-25 arcsec. The injected flux density of RHs is not fully recovered by the imaging and an increasing fraction of injected flux is lost when $\mathrm{S}_{R H}$ decreases, and/or the total angular size increases; an example is given in Fig. 2. We also found very little dependence on the resolution of the image, at least in the range $15^{\prime \prime}-25^{\prime \prime}$. We find that the lowest value of the injected flux density that leaves a residual flux in the images which can be reasonably interpreted as due to an extended low brightness radio source on the basis of the standard radio imaging is in the range $\approx 5-12 \mathrm{mJy}$. This marks the value of the upper limit to the injected flux of RHs and scales both with the largest angular size of the fake RHs and with the r.m.s of the final image (e.g., Fig. 3). Note however, that at this point a residual flux is still formally detected at $4-5 \sigma$ level on an area of a few beams in the low resolution images (e.g., Fig. 2). Our limits should thus be considered as conservative; they are typically $\approx 2.5$ times larger than those in Eq.(1). 
We derive these solid limits to the detection of RHs for 20 clusters observed at the GMRT. Indeed, among the observed 29 clusters with no hint of cluster-scale emission, we excluded A 3444, A 1682 and Z 7160 where extended radio galaxies in the field makes difficult to straightaway apply our procedure, and also excluded 6 clusters in our sample with poor quality of the data (r.m.s. $>120 \mu \mathrm{Jy} / \mathrm{b}$ caused by interferences).

\section{Results}

In Fig. 4 we report the distribution of clusters in the $P_{1.4}-L_{X}$ plane. Giant RHs and upper limits obtained from UVMOD simulations (Sect. 3) with $R_{H}=0.5 \mathrm{Mpc}$ are reported in magenta. These solid upper limits lie about one order of magnitude below the correlation for giant RHs. This allows us to firmly establish that the $P_{1.4}-L_{X}$ correlation (solid line in Fig. 4, from Cassano et al.2006) is real and that its lower envelope is not driven by observational biases (at least for $L_{X} \geq 5 \times 10^{44} \mathrm{erg} \mathrm{s}^{-1}$, i.e. the selection limit of our clusters).

Most importantly, we find that clusters with similar $L_{X}$ and redshift have a clear bimodal distribution. Cluster-scale radio emission at the level of presently studied RHs is not ubiquitous in galaxy clusters and only $\approx 1 / 3$ of clusters in our sample host a RH. Although no homogeneous high resolution X-ray data are still available for all our clusters, the RHs are found in dynamically disturbed systems, while clusters without RHs are either disturbed or relaxed systems (V07). Fig. 4 (green arrows) shows that even more stringent upper limits are obtained considering radio emission on cluster-core scale $\left(R_{H}=0.25 \mathrm{Mpc}\right)$.

It is challenging for secondary models to accommodate the observational picture of Fig. 4. These models would expect the cluster-scale radio emission much common and predict some general $P_{1.4}-L_{X}$ trend for all clusters with some scattering due to the effect of the different CR proton content and magnetic field among clusters (e.g. Miniati et al. 2001; Dolag 2006). Thus strong dissipation of the magnetic field in the ICM in relatively short time-scales is necessary to reconcile secondary model expectations with the data, although reconciling this dissipation with present theoretical understanding (e.g. Subramanian et al. 2006) and data (e.g. Govoni \& Feretti 2004) might be problematic. Recently Pfrommer (2007) presented numerical simulations of secondary \& shock accelerated particles. Also in this case extended synchroton emission, at least on cluster-core scale, is predicted to be common, at the level of presently known RHs, and clusters are predicted to follow relatively well defined correlations (Fig.1 in Pfrommer 2007) with merging and non merging systems lying on the upper and lower envelope of correlations, respectively. Thus similar considerations can be applied also to this scenario. 


\section{Limits on $\mathrm{CR}$ in non-radio emitting clusters}

Gamma ray observations of a number of nearby galaxy clusters limit the energy density of CR protons in these clusters to 10-20\% of the thermal energy (Pfrommer \& Ensslin 2004; Reimer 2004).

The upper limits for clusters with no RHs of our sample allow us to obtain indirect upper limits to the energy density of CR protons in these clusters. Indeed, by requiring that radio emission from secondary $\mathrm{e}^{ \pm}$is below the upper limits in Fig. 4 one gets constraints on the content of $\mathrm{CR}$ protons from which secondaries are injected.

We use the formalism in Brunetti \& Blasi (2005) to calculate the stationary spectrum of secondary pairs in the ICM. Typical limits to the CRs content are given in Fig.5 by simply assuming average values of thermal density $\left(n_{t h}=10^{-3} \mathrm{~cm}^{-3}\right)$ and magnetic field in a sphere of radius $R_{H}=0.5 \mathrm{Mpc}$ : for $\geq \mu \mathrm{G}$ fields and relatively flat spectra of the CRs our limits are about one order of magnitude deeper than present EGRET upper limits; note that our limits scale with $\left(n_{t h} / 10^{-3}\right)^{-2}$. Limits are also significantly lower than the typical CR energy content of clusters that is claimed from numerical simulations in which CRs are accelerated at large scale shocks (e.g. Ryu et al. 2003).

On the other hand, for steeper CR spectra (or lower values of the field) the synchrotron constraints become gradually less stringent and the energy content of CRs in our clusters may be considerably larger.

\section{Conclusions}

We have reported on constraints on the origin of $\mathrm{RHs}$ and on the $\mathrm{CR}$ content in the ICM obtained via radio observations of a fairly large sample of $\mathrm{X}$-ray luminous clusters at $z=0.2-0.4$.

In the bulk of these clusters we do not find evidence of $\mathrm{Mpc}$-scale radio emission at the

level of RHs. Our conclusions become even more stringent considering radio emission on cluster-core scale, typical of smaller RHs and mini-Halos.

We firmly confirm that RH-clusters follow a physical correlation between synchrotron and $\mathrm{X}$-ray luminosities. We find that clusters have a bimodal distribution in the $P_{1.4}-L_{X}$ plane (Fig. 4); this is in line with the expectation of the re-acceleration scenario. On the other hand, in order to reconcile these observations with expectations from secondary models strong dissipation of the magnetic field in the clusters with no radio emission is necessary.

Our measurements allow us to also derive simple limits on the presence of CR protons in 
the ICM (Fig. 5). In the case of relatively flat spectral energy distribution of these CRs stringent upper limits can be obtained: the energy density of CRs should be $\leq 1 \%$ of the thermal energy in case of $\geq \mu \mathrm{G}$ field strength. This would make problematic the detection of gamma rays from $\pi^{o}$-decay in clusters with GLAST. On the other hand, by assuming steeper

spectral energy distributions of these CRs (or lower magnetic fields) our limits become less stringent.

\section{Acknowledgements}

We acknowledge M.Bondi and an anonymous referee for useful comments, and support through grants ASI-INAF I/088/06/0 and PRIN-MUR 2006-02-5203.

\section{REFERENCES}

Bacchi M., Feretti L., Giovannini G., Govoni F., 2003, A\&A 400, 465

Blasi P., Colafrancesco S., 1999, APh 12, 169

Blasi P., Gabici S., Brunetti G., 2007, IJMPA 22, 681

Böhringer H., Schuecker P., Guzzo L., et al., 2004, A\&A 425, 367

Brunetti G., Setti G., Feretti L., Giovannini G., 2001, MNRAS 320, 365

Brunetti G., Blasi P., Cassano R., Gabici S., 2004, MNRAS 350, 1174

Brunetti G., Blasi P., 2005, MNRAS 363, 1173

Brunetti G., Lazarian A, 2007, MNRAS 378, 245

Cassano R., Brunetti G., 2005, MNRAS 357, 1313

Cassano R., Brunetti G., Setti G., 2006, MNRAS 369, 1577

Cassano R., Brunetti G., Setti G., Govoni F., Dolag K., 2007, MNRAS 378, 1565

Clarke T.E., 2005, ASP Conference Series, Eds. by N.E. Kassim, M.R. Perez, W.Junor and P.A. Henning, Vol 345, pag. 227

Clarke T.E., Kronberg P.P., Böringer H., 2001, ApJ 547, 111 
Ebeling H., Edge A.C., Böringer H., et al., 1998, MNRAS 301, 881

Ebeling H., Edge A.C., Allen S.W., Crawford C.S., Fabian A.C., Huchra J.P., 2000, MNRAS 318,333

Feretti L., Brunetti G., Giovannini G., et al., 2004, JKAS 37, 315

Feretti L., Giovannini G., 2007, arXiv:astro-ph/0703494

Gabici S., Blasi P., 2003, ApJ 583, 695

Giacintucci S., Venturi T., Bardelli S., Brunetti G., Cassano R., Dallacasa D., 2006, NewA 11,437

Giovannini G., Tordi M., Feretti L., 1999, NewA 4, 141

Govoni F., Feretti L., 2004, IJMPD 13, 1549

Kempner J.C., Sarazin C.L., 2001, ApJ 548, 639

Liang H., Hunstead R.W., Birkinshaw M., Andreani P., 2000, ApJ 544, 686

Miniati F., Jones T.W., Kang H., Ryu D., 2001, ApJ 562, 233

Dolag K., 2006, AN 327, 575

Dolag K., Ensslin T.A., 2000, A\&A 362, 151

Petrosian V., 2001, ApJ 557, 560

Pfrommer C., 2007, sumbitted to MNRAS, arXiv:0707.1693

Pfrommer C., Ensslin T.A., 2004, A\&A 413, 17

Pfrommer C., Springel V., Ensslin T.A., Jubelgas M., 2006, MNRAS 367, 113

Reimer O., 2004, JKAS 37, 307

Rudnick L., Delain K.M., Lemmerman J.A., 2006, AN 327, 549

Ryu D., Kang H., Hallman E., Jones T.W., 2003, ApJ 593, 599

Sarazin C.L., 1999, ApJ 520, 529

Subramanian K., Shukurov A., Haugen N.E.L., 2006, MNRAS 366, 1437 
Venturi T., Giacintucci S., Brunetti G., Cassano R., Bardelli S., Dallacasa D., Setti G., 2007, A\&A 463, 937

Völk H.J., Atoyan A.M., 1999, APh 11, 73 


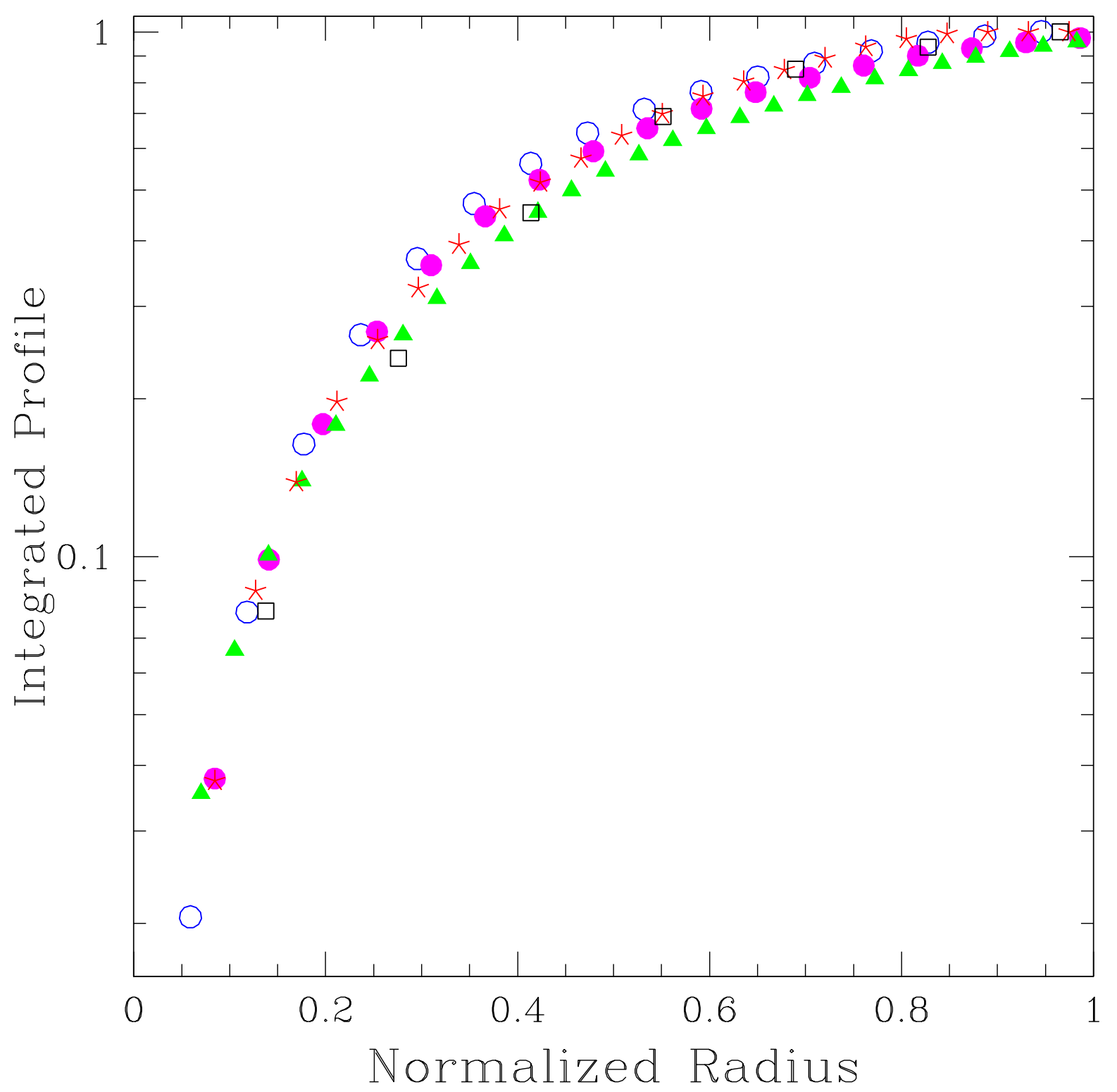

Fig. 1. - Integrated brightness profiles of well studied RHs (from Cassano et al. 2007): A545 (boxes), A2319 (triangles), A2744 (empty circles), A2163 (filled circles), A2255 (stars). 

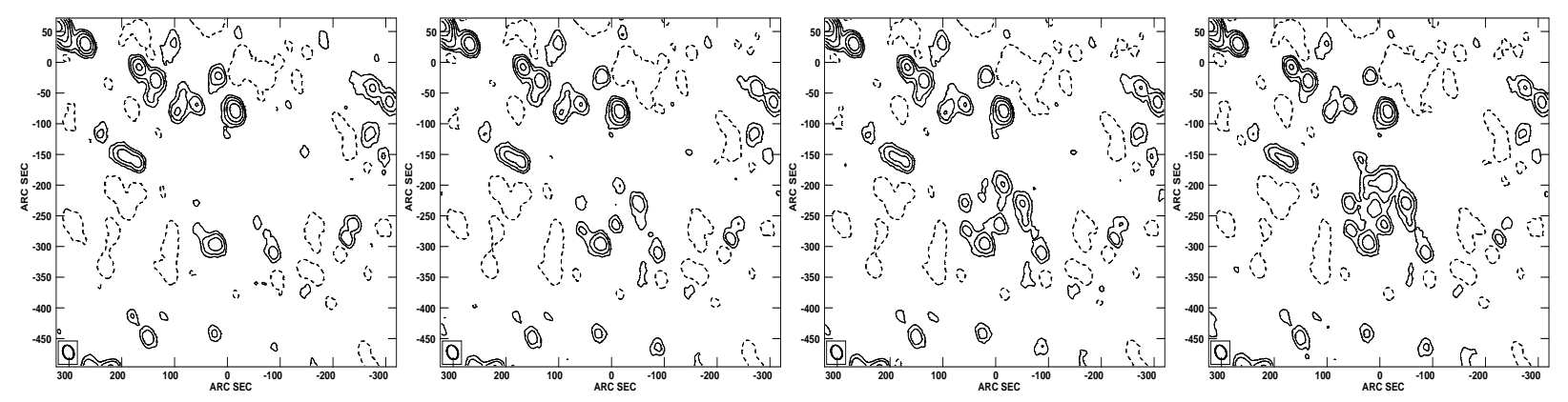

Fig. 2. - Example of injection of fake RHs with apparent radius $\Theta_{H}=150 \operatorname{arcsec}$ and $S_{R H}=$ $0,8,11$ and $15 \mathrm{mJy}$ (from left to right). The r.m.s. of the image is $65 \mu \mathrm{Jy} / \mathrm{b}\left(\theta_{b} \simeq 20 \times 24\right.$ arcsec). Contour levels are given for $0.1 \times(-1,1,2,4,8,16,32,64) \mathrm{mJy} / \mathrm{b}$. In this case diffuse emission is revealed with standard analysis (including comparison between high and low resolution images) for $S_{R H}>11 \mathrm{mJy}$. 


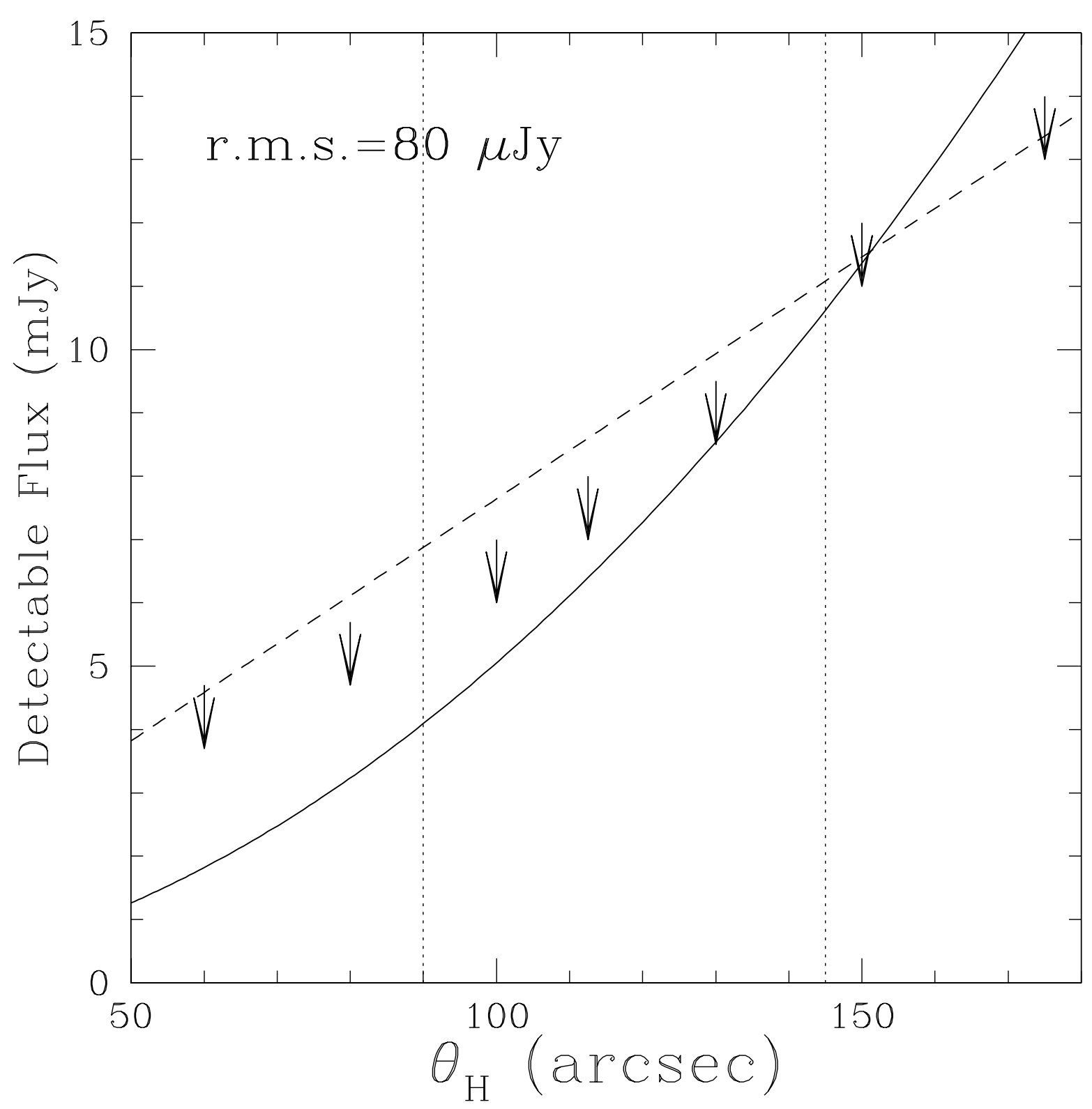

Fig. 3.- Upper limits on the detectable RH flux (arrows) from UVMOD simulations (GMRT cluster field with r.m.s. $=80 \mu \mathrm{Jy} /$ beam and beam $=20 \times 22 \mathrm{arcsec}$ ) as a function of apparent radius $\Theta_{H}$ (in arcsec). The solid line marks the constant brightness scaling, the dashed line marks the $1 / \Theta_{H}$ scaling. The vertical dotted lines mark the range of $\Theta_{H}$ spanned by our clusters $\left(R_{H}=0.5 \mathrm{Mpc}\right)$. 


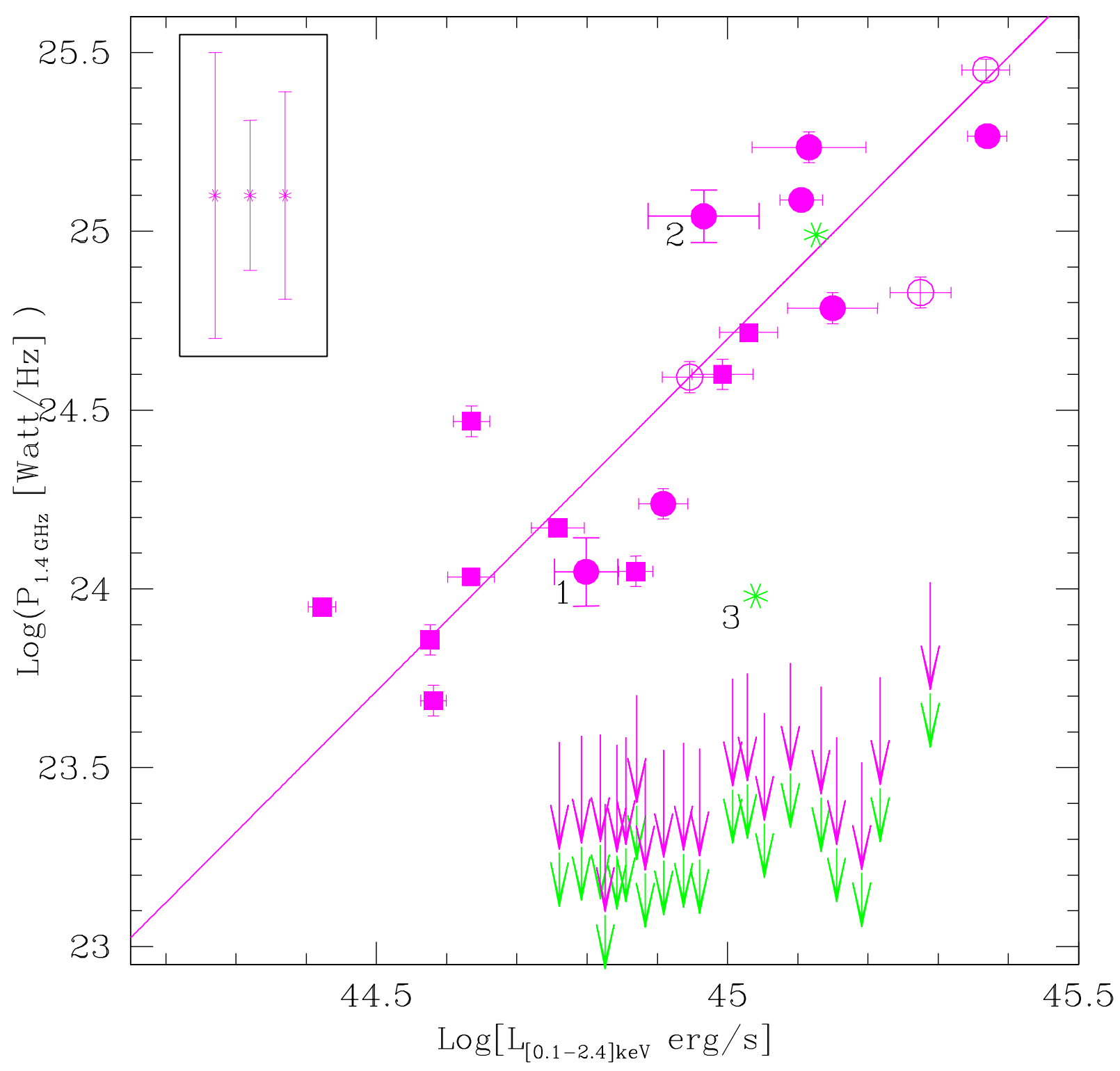

Fig. 4.- Clusters in the $P_{1.4}-L_{X}$ diagram: published giant RHs in our sample (magenta filled circles), other giant RHs at $z>0.2$ (magenta empty circles) and at $z<0.2$ (magenta filled squares). Upper limits are obtained assuming $R_{H}=0.5$ (magenta) and $0.25 \mathrm{Mpc}$ (green) and are scaled at $1.4 \mathrm{GHz}$ with a typical spectral index of RHs, $\alpha=1.3$ (Feretti et al. 2004; $\left.P(\nu) \propto \nu^{-\alpha}\right)$. The mini-Halo A2390 and the small RH RXJ1314 which are in our sample are also reported (green asterisks). RHs taken from GMRT observations are: 1=A209, 2= RXJ2003, 3=RXJ1314 (TV07). Estimated dispersions in $P_{1.4}$ at fixed cluster mass/temperature from simulations of secondary models are reported (from left to right: Miniati et al. 2001, Dolag \& Ensslin 2000, Pfrommer 2007). 


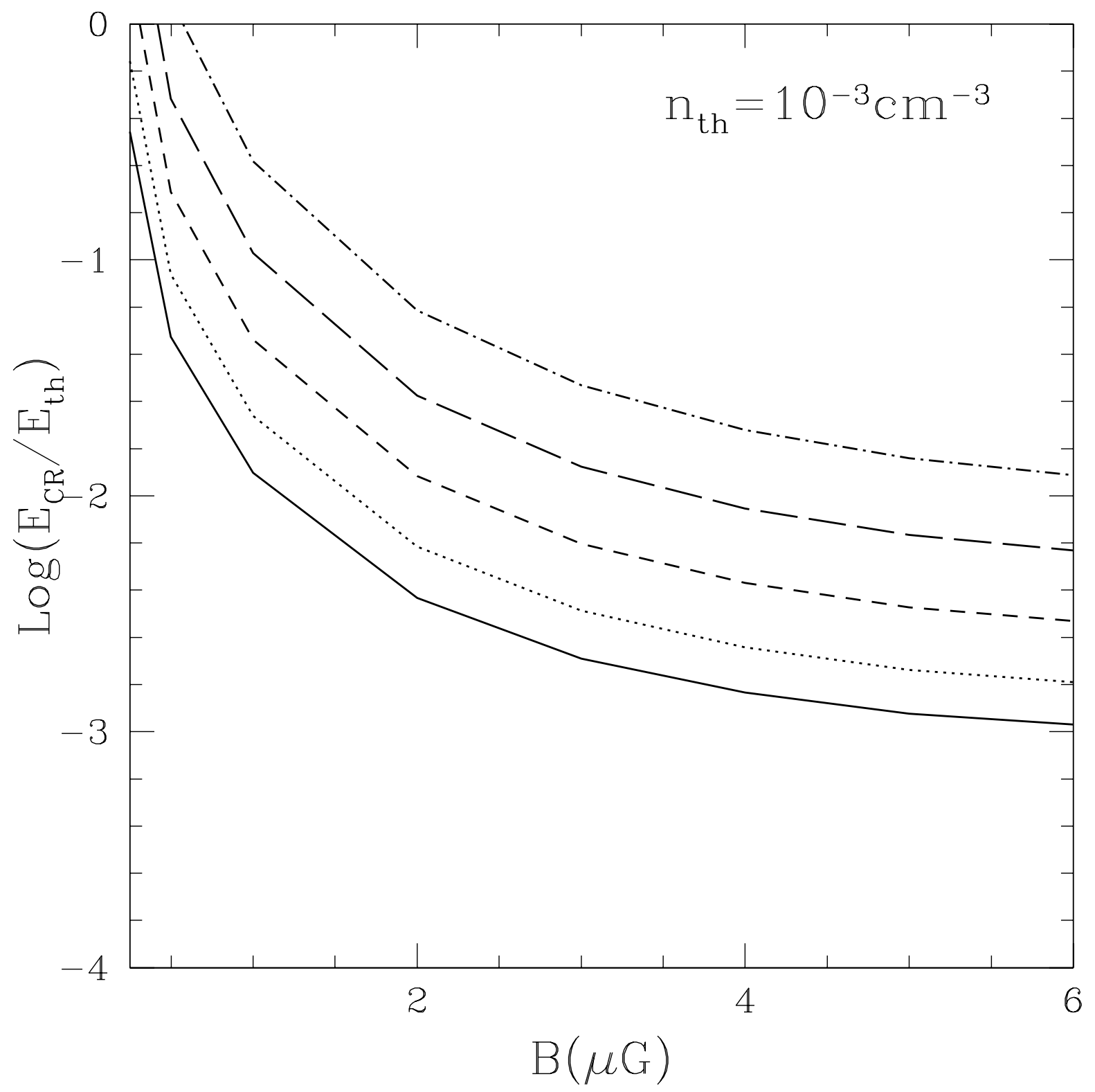

Fig. 5.- Upper limits (curves) to the energy density (for $p>0.01 m_{p} c$ ) of CRs (normalised to the thermal energy density) as a function of the magnetic field. We assume a CRs spectrum $\propto p^{-\delta}(\delta=2.1,2.3,2.5,2.7,2.9$ from bottom to top), $z=0.25$ (for inverse Compton losses), thermal density $=10^{-3} \mathrm{~cm}^{-3}$, temperature $=10^{8} \mathrm{~K}$ and synchrotron upper limits $=10^{24} \mathrm{~W} / \mathrm{Hz}$ at $610 \mathrm{MHz}$. 